\title{
BROWN-PETERSON AND ORDINARY COHOMOLOGY THEORIES OF CLASSIFYING SPACES FOR COMPACT LIE GROUPS
}

\author{
AKIRA KONO AND NOBUAKI YAGITA \\ Dedicated to Professor Tokushi Nakamura on his 60th birthday
}

\begin{abstract}
The Steenrod algebra structures of $H^{*}(B G ; Z / p)$ for compact Lie groups are studied. Using these, Brown-Peterson cohomology and Morava $K$ theory are computed for many concrete cases. All these cases have properties similar as torsion free Lie groups or finite groups, e.g., $B P^{\text {odd }}(B G)=0$.
\end{abstract}

\section{INTRODUCTION}

Let $B G$ be the classifying space of a compact Lie group $G$. Let $p$ be a fixed prime. It is well known that if $H^{*}(B G)_{(p)}$ has no $p$-torsion, then it is a polynomial algebra generated by even dimensional elements. Therefore the Atiyah-Hirzebruch type spectral sequence converging to the Brown-Peterson cohomology $B P^{*}(B G)$ collapses and $B P^{*}(B G) \simeq B P^{*} \otimes H^{*}(B G)_{(p)}$ where $\otimes$ denotes completed tensor product (see $\S 1$ ). Hence we get:

(1) $B P^{*}(B G)=B P^{\text {even }}(B G)$.

(2) $B P^{*}(B G)$ is $p$-torsion free.

(3) $B P^{*}(B G)$ has no nilpotent elements.

(4) $B P^{*}(B G)$ is $B P^{*}$-flat for finite $B P^{*}(B P)$-modules. Moreover

$$
B P^{*}\left(B G \times B G^{\prime}\right) \simeq B P^{*}(B G) \otimes_{B P^{*}} B P^{*}\left(B G^{\prime}\right)
$$

for all compact Lie groups $G^{\prime}$.

(5) $K(n)^{*}(B G) \simeq K(n)^{*} \otimes_{B P^{*}} B P^{*}(B G)$

where $K(n)^{*}(-)$ is the Morava $K$-theory. Moreover if $G$ is a classical Lie group, we know

(6) $B P^{*}(B G)=\mathrm{Ch}_{B P}(B G)$, the Chern subring of $B P^{*}(B G)$ generated by Chern classes for all complex representations.

The main purpose of this paper is to show that the above properties hold in many cases even if $H^{*}(B G)$ has $p$-torsion. Note that for the ordinary cohomology theory $H^{*}(B G)_{(p)}$, the corresponding properties (1)-(4), (6) do not always hold, for example, $H^{*}(B G)_{(p)} \neq H^{\text {even }}(B G)_{(p)}$. Landweber showed (1)(6) hold for all abelian groups [L1]. Moreover he conjectured (2), (4), (6) for

Received by the editors July 12, 1991.

1991 Mathematics Subject Classification. Primary 57R77; Secondary 55N22.

Key words and phrases. Classifying space $B G$, compact Lie groups, BP-theory, cohomology operations. 
all compact Lie groups in [L3]. By [T-Y, Y2] when $G$ is a direct product of metacyclic groups or minimal nonabelian p-groups (1)-(6) hold. A result of Hopkins, Kuhn and Ravenel [H-K-R] easily shows that when $G$ is a finite group, (2) implies

$$
B P^{*}(B G) \hookrightarrow \lim _{\longleftarrow} B P^{*}(B A),
$$

$A$ runs through all conjugacy classes of abelian subgroups of $G$.

Remark that if a $p$-Sylow subgroup of a finite group $G$ satisfies (1)-(7), so does $G$.

On the other hand, Wilson showed that $B P^{*}(B O(n))$ is generated by the Chern classes of the complexification of the universal real bundle. By using Wilson's arguments, we show

Theorem 1. Properties (1)-(2) and properties (4)-(6) hold for direct products of $O(n), S O(2 n+1)$.

The ordinary cohomology rings $H^{*}(B G ; Z / p)$ for $G=F_{4}, P U(3)$ are given by Toda [T1] and by Kono, Mimura, and Shimada [K-M-S]. We study $H^{*}(B P U(3))$ in detail, considering the relation to its abelian subgroups. Hence we get

$$
H^{*}(B G ; Z / p) \hookrightarrow \lim H^{*}(B A ; Z / p)
$$

for $G=P U(3)$. This was conjectured by J. F. Adams for all connected compact Lie groups $G$ and $p \geq 3$ and solved for $G=F_{4}, p=3$ by Adams and Kono. We also know that there are only two conjugacy classes of maximal elementary 3abelian subgroups of $P U(3)$. Moreover we can determine the Steenrod algebra structure of $H^{*}(B P U(3))$. Using these, we show

Theorem 2. Properties (1)-(5), (7) hold when $G=P U(3)$ and $F_{4}$ for $p=3$, but (6) does not hold for $G=P U(3)$.

Mimura and Kono study $H^{*}(B G ; Z / p)$ for many compact Lie groups [K$M 1,2]$. Also using their results, we get

Theorem 3. The properties (1)-(3), (7) hold when $G=\operatorname{Spin}(n) n \leq 10, G_{2}, F_{4}$, $E_{6}, P S U(4 n+2)$ for $p=2$.

Bakuradze [B-N] showed that (1)-(7) hold for the normalizer group of maximal torus in $S p(1) \times S p(1)$. Hunton showed $K(n)^{*}(B G)=K(n)^{\text {even }}(B G)$ for some other compact Lie groups $[\mathrm{H}]$. Inoue [I] determined $B P^{*}(B S O(6))$ and showed (1) for this case.

Conjecture 4. Assertions (1)-(5) and (7) hold for all compact Lie groups.

There are no application of these results now. However we hope $B P^{*}(B G)$ can aid in the understanding of the ordinary cohomology $H^{*}(B G)$ which seems so complicated in general cases. For example, we presume that the following conjecture, which holds in all cases in Theorems 1-3, is true.

Conjecture 5. If $G$ is a connected compact Lie group, then for each odd dimensional element $x \in H^{*}(B G ; Z / p)$, there is $i$ such that $Q_{m} x \neq 0$ for all $m \geq i$, where $Q_{m}$ are the Milnor primitive operators. 
The authors would like to thank Norihiko Minami and Michisige Tezuka for many conversations and Koemon Irie who pointed out errors in the first version.

\section{BP AND RELATED COHOMOLOGY THEORIES}

Throughout this paper, we assume that spaces $X, Y$ mean $C W$-complexes whose $n$-skeleton is finite complexes for each $n \geq 0$. Let $B P^{*}(-)$ be the Brown-Peterson cohomology localized at $p$ with the coefficient $B P^{*}=$ $Z_{(p)}\left[v_{1}, \ldots\right],\left|v_{i}\right|=-2\left(p^{i}-1\right)$. We consider the cohomology theories $k^{*}(-)$ with the coefficient $k^{*}=B P^{*} /($ Ideal $S)$, e.g., $P(n)^{*}=Z / p\left[v_{n}, v_{n+1}, \ldots\right]$, $\widetilde{P}(n)^{*}=Z_{(p)}\left[v_{n}, \ldots\right], B P\langle n\rangle^{*}=Z_{(p)}\left[v_{1}, \ldots, v_{n}\right], k(n)^{*}=Z / p\left[v_{n}\right], \tilde{k}(n)^{*}=$ $Z_{(p)}\left[v_{n}\right]$, and $K(n)^{*}=Z / p\left[v_{n}, v_{n}^{-1}\right]$. We consider the Atiyah-Hirzebruch spectral sequence $E_{2}^{*}, *=H^{*}\left(X ; k^{*}\right) \Rightarrow k^{*}(X)$. Hereafter we assume the convergence of this spectral sequence and hence

$$
j^{*}: k^{*}(X) \simeq \underset{N}{\lim } k^{*}\left(X^{N}\right)
$$

Note that if $X=B G$ or $k^{n}$ is a fine group, this assumption holds. (See [L3].)

Define a filtration $F^{N}(X)=\operatorname{Ker}\left(j_{N}^{*}: k^{*}(X) \rightarrow k^{*}\left(X^{N}\right)\right)$ of $k^{*}(X)$ and define a topology in $k^{*}(X)$ by $F^{N}(X)$ as the fundamental neighbourhoods of 0 . Then

$$
k^{*}(X) \simeq \underset{N}{\lim } k^{*}(X) / F^{N}(X)
$$

is a complete algebra. Let $A, B$ be $k^{*}$-complete algebras with filtrations $A^{N}$, $B^{N}$. We define the complete tensor product $\otimes$ by

$$
A \otimes_{k^{*}} B \simeq \underset{\lim }{\longleftarrow} \tilde{\otimes}_{k^{*}} B /\left(A^{N} \tilde{\otimes}_{k^{*}} B+A \tilde{\otimes}_{k^{*}} B^{N}\right)
$$

where $\tilde{\otimes}$ is the usual tensor product. Then if $X$ and $Y$ are $p$-torsion free, then we can write

$$
k^{*}(X \times Y) \simeq k^{*}(X) \otimes_{k^{*}} k^{*}(Y) .
$$

Note that $-\otimes_{k^{*}}-$ in this paper means that each element is expressed as infinite sum.

Landweber's exact functor theorem [L2] says that injectivity of the following (1.5) for all $n \geq 0$ (let $p=v_{0}$ ),

$$
v_{n}: P(n)^{*} \otimes_{B P^{*}} B P^{*}(X) \rightarrow P(n)^{*} \otimes_{P(n)^{*}} B P^{*}(X)
$$

implies the $B P^{*}$-flatness of $B P^{*}(X)$ for finite $B P^{*}(B P)$-modules. In particular we have

$$
B P^{*}(X \times Y) \simeq B P^{*}(X) \otimes_{B P^{*}} B P^{*}(Y) \text { for all } Y \text { satisfies (1.1). }
$$

From the Sullivan exact triangles

$$
\begin{aligned}
& B P^{*}(X) \stackrel{\rho}{\longrightarrow} P(1)^{*}(X) \stackrel{\rho}{\longrightarrow} P(2)^{*}(X) \stackrel{\rho}{\longrightarrow} P(3)^{*}(X) \cdots \\
& p \backslash \delta \swarrow \quad v_{1} \backslash \quad \delta \swarrow \quad v_{2} \backslash \quad \swarrow \delta \\
& B P^{*}(X) \quad P(1)^{*}(X) \quad P(2)^{*}(X)
\end{aligned}
$$

the injectivity of $(1.5)$ is equivalent to the assertion that $\rho: B P^{*}(X) \rightarrow P(n)^{*}(X)$ is epic for all $n \geq 0$ and is that

$$
P(n)^{*}(X) \simeq P(n)^{*} \otimes_{B P^{*}} B P^{*}(X) .
$$


From Johnson-Wilson theorem [J-W], if $X$ satisfies (1.7), then we get

$$
K(n)^{*}(X) \simeq K(n)^{*} \otimes_{B P^{*}} B P^{*}(X) .
$$

Lemma 1.9. If $X$ and $Y$ satisfy the injectivity of (1.5), then so does $X \times Y$.

Proof. By the exact functor theorem for $P(n)^{*}$-theory, we have

$$
P(n)^{*}(X \times Y) \simeq P(n)^{*}(X) \otimes_{P(n)^{*}} P(n)^{*}(Y),
$$

which is isomorphic to

$$
P(n)^{*} \otimes_{B P^{*}}\left(B P^{*}(X)\right) \otimes_{P(n)^{*}} P(n)^{*} \otimes_{B P^{*}}\left(B P^{*}(Y)\right) \simeq P(n)^{*} \otimes_{B P^{*}} B P^{*}(X \times Y) .
$$

Therefore $X \times Y$ satisfies (1.7) and so (1.5).

By the same argument as Theorem 3.3 [Y2], the kernel of $r: B P^{*}(B G) \rightarrow$

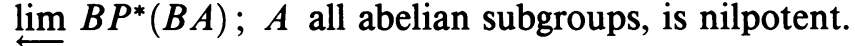

Lemma 1.10. The injectivity of $r$ is equivalent to that $B P^{*}(B G)$ has no nonzero nilpotent element.

Proof. We only need $B P^{*}(B A)$ has no nilpotent element. Consider the spectral sequence induced from

$$
0 \rightarrow A^{\prime} \rightarrow A \rightarrow Z / p \rightarrow 0
$$

Then

$$
E_{2}^{*, *^{\prime}} \simeq E_{\infty}^{*, *^{\prime}}=H^{*}(Z / p) \otimes B P^{*^{\prime}}\left(A^{\prime}\right) /(p) \simeq Z / p[y] \otimes B P^{*^{\prime}}\left(A^{\prime}\right)
$$

for ${ }^{*}>0$, since $B P^{*}\left(A^{\prime}\right)$ is $p$-torsion free. Suppose $a^{l}=0$ in $B P^{*}(B A)$.

Let $y^{s} x \neq 0 \in E_{\infty}^{2 s,}$ be the corresponding element to $a$. Since $\left(y^{s} x\right)^{l}=$ $0, x^{l}=0$ in $B P^{*}\left(B A^{\prime}\right) /(p)$. Let us write $x^{l}=p^{r} x^{\prime}, x^{\prime} \neq 0 \bmod p$ in $B P^{*}\left(B A^{\prime}\right)$. Then

$$
a^{l}=p^{r} x^{\prime} y^{s}=v_{1}^{r} y^{s+r(p-1)} x^{\prime} \quad \text { in } E_{\infty}^{2(s+r(p-1)), *} .
$$

This element is nonzero because $B P^{*}\left(B A^{\prime}\right) /(p)$ has no $v_{1}$-torsion.

Therefore we get the following implications:

$$
\begin{aligned}
& \text { (3) } \Leftrightarrow(7) \Rightarrow(2) \Leftarrow((1.7) \text { for } X=B G) \Leftrightarrow(4) \Rightarrow(5) \\
& (1) \Leftarrow(6) \text {. }
\end{aligned}
$$

Kuhn, Hopkins, and Ravenel showed that when $G$ is a finite group, $|G|^{-1} r$ is isomorphic. Therefore

$$
\text { (2) } \Leftrightarrow(7) \text { for finite groups. }
$$

\section{THE ORTHOGONAL GROUP $O(n)$}

Before considering $B P^{*}(B O(n))$, we consider cohomology operations $Q_{i}$. Recall $Q_{i+1}=\mathscr{P}^{p^{i}} Q_{i}-Q_{i} \mathscr{P} p^{i}\left(=\mathrm{Sq}^{2^{i}} Q_{1}+Q_{i} \mathrm{Sq}^{2^{i}}\right.$ for $\left.p=2\right)$ and $Q_{0}=$ $\mathscr{B}\left(Q_{0}=\mathrm{Sq}^{1}\right)$. The first nonzero differential of the spectral sequence

$$
E_{2}^{* * *}=H^{*}\left(X ; P(m)^{*}\right) \Rightarrow P(m)^{*}(X)
$$

is given by $d_{2 p^{m}-1}=v_{m} \otimes Q_{m}$. 
Lemma 2.2. Let $E_{i}=\Lambda\left[Q_{m}, \ldots, Q_{m+i-1}\right]$ and $E_{0}=Z / p$. Suppose that there is an $E_{i}$-module injective $E_{i} \otimes G_{i} \subset H^{*}(X ; Z / p)$ and there is a $Z / p$-module isomorphism

$$
H^{*}(X ; Z / p) \simeq \bigoplus_{i=0}^{M} E_{i} \otimes G_{i}
$$

such that $Q_{m} \cdots Q_{m+i-1} G_{i} \in \operatorname{Im} \rho\left(P(m)^{*}(X) \rightarrow H^{*}(X ; Z / p)\right)\left(\right.$ for $i=0 G_{0} \in$ $\operatorname{Im} \rho)$. Then the infinite term of the spectral sequence $(2.1)$ is

$$
E_{\infty}^{*, *} \simeq \bigoplus_{i>0}^{M} P(i+m)^{*} Q_{m} \cdots Q_{m+i-1} G_{i} \oplus P(m)^{*} G_{0} .
$$

Proof. By the induction on $r$ for $d_{2 p^{m+r-1}-1}$, we assume that $E_{2 p^{m+r-1}}=A_{r} \oplus B_{r}$, where

$$
\begin{aligned}
& A_{r}=\bigoplus_{i=0}^{r} P(m+i)^{*} Q_{m} \cdots Q_{m+i-1} G_{i}, \\
& B_{r}=\bigoplus_{i=r+1}^{M} P(m+r)^{*} Q_{m} \cdots Q_{m+r-1}\left(E_{m+r, i-r}\right) G_{i},
\end{aligned}
$$

and where $E_{m+r, i-r}=\Lambda\left[Q_{m+r}, \ldots, Q_{m+i-1}\right]$. Indeed, $A_{0}=P(n)^{*} G_{0}$ and $B_{0}=P(m)^{*} \otimes\left(\bigoplus_{i=1}^{M} E_{i} \otimes G_{i}\right)$, hence $A_{0} \oplus B_{0}=P(m)^{*} \otimes H^{*}(X ; Z / p)$.

By the supposition of the lemma, all elements in $A$ are infinite cycles. Assume $d_{s} x \neq 0, x \in B$. Since $B$ is a $P(m+r)^{*}$-free modules, it is necessary $s \geq 2 p^{m+r}-1$. Hence consider $d_{2 p^{m+r}-1}=v_{m+r} \otimes Q_{m+r}$. Therefore

$$
\begin{aligned}
E_{2 p^{m+r}} & \simeq A_{r} \oplus \bigoplus_{i=r+1}^{M} P(m+r+1)^{*} Q_{m} \cdots Q_{m+r}\left(E_{m+r+1, i-r-1}\right) G_{i} \\
& \simeq A_{r+1} \oplus B_{r+1} .
\end{aligned}
$$

Since $B_{M+1}=0$, we get the lemma.

If $H^{*}(X ; Z / p)=E_{0, n}=\Lambda\left(Q_{0}, \ldots, Q_{n}\right)$, then

$$
P(m)^{*}(X) \simeq P(n)^{*} \Lambda\left[Q_{0}, \ldots, Q_{m-1}\right] .
$$

This fact is known as $X=V(n)$, Smith-Toda spectrum.

The $B P$-cohomology of the classifying space of the $n$th orthogonal group, $B P^{*}(B O(n))$ is computed by W. S. Wilson. Since $H^{*}(B O(n))$ has only 2torsion, we need only consider the 2-primary part.

Theorem 2.3 (Wilson [W]).

$$
B P^{*}(B O(n)) \simeq B P^{*}\left[\left[c_{1}, \ldots, c_{n}\right]\right] /\left(c_{1}-c_{1}^{*}, \ldots, c_{n}-c_{n}^{*}\right)
$$

where $c_{i}$ is the ith Chern class of complexification of universal real bundle and $c_{i}^{*}$ is the Chern class of its complex conjugation.

Recall the $Z / 2$-cohomology of $B O(n)$ and $(B Z / 2)^{n}$. It is well known

$$
\begin{gathered}
H^{*}(B O(n) ; Z / 2) \hookrightarrow H^{*}\left((B Z / 2)^{n} ; Z / 2\right) \\
\text { t\| } \| \\
Z / 2\left[w_{1}, \ldots, w_{2}\right] \hookrightarrow Z / 2\left[x_{1}, \ldots, x_{n}\right]
\end{gathered}
$$

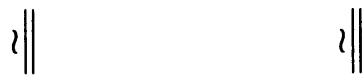


where $w_{i}$ is the $i$ th elementary symmetric polynomial of $x_{s}$. Then $w_{i}$ is the $i$ th Whitney class and $c_{i}=i^{*}\left(c_{i}\right)=w_{i}^{2}$ for the complexification map $i: B O(n) \rightarrow B U(n)$. The following lemma is just the $P(m)^{*}$-analogue of Wilson's (p. 359, Theorem 2.1 in [W]).

Lemma 2.4. Let $G_{k}$ be $Z / 2$-vector space in $H^{*}(B O(n) ; Z / 2)$ generated by symmetric functions

$$
\sum x_{1}^{2 i_{1}+1} \cdots x_{k}^{2 i_{k}+1} x_{k+1}^{2 j_{1}} \cdots x_{k+q}^{2 j_{q}}, \quad k+q \leq n,
$$

with $0 \leq i_{1} \leq \cdots \leq i_{k}$ and $0 \leq j_{1} \leq \cdots \leq j_{q}$; and if the number of $j$ equal to $j_{u}$ is odd, then there is some $s \leq k$ such that

$$
2 i_{s}+2^{s+m}<2 j_{u}<2 i_{s}+2^{s+m+1} .
$$

Then $G_{k}$ satisfies the assumption of Lemma 2.2 and hence the infinite term of the Atiyah-Hirzebruch spectral sequence converging to $P(m)^{*}(B O(n))$ is

$$
E_{\infty}^{*, *} \simeq \bigoplus_{i=0}^{n} P(m+r)^{*} Q_{m} \cdots Q_{m+r} G_{r}
$$

Proof. First note $Q_{m} \cdots Q_{m+r} G_{r}$ is generated by functions of $\sum x_{1}^{2 h_{1}} \cdots x_{k+q}^{2 h_{k+q}}$ and hence is in $Z / 2\left[w_{1}^{2}, \ldots, w_{n}^{2}\right]$ which is in

$$
\left.\operatorname{Im} \rho\left(P(m)^{*} B O(n)\right) \rightarrow H^{*}(B O(n) ; Z / 2)\right)
$$

since $i^{*}\left(c_{j}\right)=w_{j}^{2}$. The proof for satisfying the assumption of Lemma 2.2 is completely the same as the proof of Wilson's Theorem 1 [W, p. 360] except for changing $Q_{i}$ to $Q_{m+i}$ and $2^{v+1}$ to $2^{v+m}$.

\section{Corollary 2.5 .}

$$
\begin{aligned}
P(m)^{*}(B O(n)) & \simeq P(m)^{*} \otimes_{B P^{*}} B P^{*}(B O(n)), \\
K(m)^{*}(B O(n)) & \simeq K(m)^{*} \otimes_{B P^{*}} B P^{*}(B O(n)) .
\end{aligned}
$$

Proof. From Lemma 2.4, $\rho: B P^{*}(B O(n)) \rightarrow P(m)^{*}(B O(n))$ is epic.

It is well known that there is an isomorphism of Lie groups

$$
Z / 2 \times S O(2 n+1) \simeq O(2 n+1)
$$

and hence

$$
B P^{*}(B Z / 2) \otimes_{B P^{*}} B P^{*}(B S O(2 n+1)) \simeq B P^{*}(B O(2 n+1)),
$$

and $B P^{*}(B Z / 2)$ is $B P^{*}$-flat. Therefore $B P(B S O(2 n+1))$ is generated by $c_{i}\left(=w_{i}^{2}\right), 2 \leq i \leq 2 n+1$. The same facts hold for $P(m)^{*}$-theory $n \geq 1$. Hence $\rho: B P^{*}(B S O(2 n+1)) \rightarrow P(m)^{*}(B S O(2 n+1))$ is epic. Therefore we get Theorem 1 in the introduction.

Remark that the squaring operation is given by

$$
\mathrm{Sq}^{i} w_{k}=\sum_{j}^{i}\left(\begin{array}{c}
k-j-1 \\
i-j
\end{array}\right) w_{k+i-j} w_{j} \quad(0 \leq i \leq k) .
$$




\section{3. Сономоlogy of $B P U(3)$}

The projective unitary group $P U(3)$ is defined as $P U(3)=S U(3) / \Gamma$ where $\Gamma$ is the center of $S U(3)$. Let us write

$$
\tilde{a}=\left(\begin{array}{ccc}
0 & 1 & 0 \\
0 & 0 & 1 \\
1 & 0 & 0
\end{array}\right), \quad \tilde{b}=\left(\begin{array}{ccc}
w & 0 & 0 \\
0 & w^{2} & 0 \\
0 & 0 & 1
\end{array}\right), \quad \tilde{c}=\left(\begin{array}{ccc}
w & 0 & 0 \\
0 & w & 0 \\
0 & 0 & w
\end{array}\right)
$$

where $w=\exp (2 \pi i / 3)$. Note that $\Gamma=\langle\tilde{c}\rangle$. The group generated by $\langle\tilde{a}, \tilde{b}, \tilde{c}\rangle$ is $E$, the nonabelian 3-group of order 27 with its exponent 3. Consider the elementary abelian 3-subgroups $V_{1}=E / \Gamma=\langle\tilde{a}\rangle \oplus\langle\tilde{b}\rangle$ and $V_{2} \subset T^{2}$, the maximal torus of $P U(3)$.

Quillen [Q1] proved that for a compact Lie group

$$
r: H^{*}(B G ; Z / p) \simeq \underset{V}{\lim _{V}} H^{*}(B V ; Z / p)
$$

is an $F$-isomorphism, where $V$ runs the conjugacy classes of elementary $p$ subgroups of $G$. Here an $F$-isomorphism means $\operatorname{Ker} r \subset \sqrt{0}$; nilpotent elements and for each $x, x^{p^{s}} \in \operatorname{Im} r$ for some $s$.

We will prove a much stronger result for $G=P U(3)$.

Theorem 3.3. The restriction map

$$
r: H^{*}(B P U(3) ; Z / 3) \rightarrow H^{*}\left(B V_{1} ; Z / 3\right) \otimes H^{*}\left(B V_{2} ; Z / 3\right)
$$

is injective.

Therefore $\left\{V_{1}, V_{2}\right\}$ is the set of the conjugacy classes of maximal elementary 3-subgroups in $P U(3)$.

Let $\rho$ be the canonical representation in $S U(3)$ and

$$
\tilde{\lambda}=\rho \otimes \rho^{-1}: S U(3) \rightarrow S U(9) .
$$

Since $\rho \otimes \rho^{-1} \mid \Gamma$ is trivial, $\tilde{\lambda}$ induces the representation $\lambda$ of $P U(3)$. It is easily computed.

\section{Lemma 3.5.}

$$
\chi_{\tilde{\lambda}}\left(a^{i} b^{j}\right)= \begin{cases}9, & i \equiv 0, j \equiv 0 \bmod 3, \\ 0, & \text { otherwise. }\end{cases}
$$

Corollary 3.6. $\lambda \mid V_{1}$ is the regular representation.

Think of $P U(3)$ as $U(3) / \widetilde{\Gamma}$ where $\widetilde{\Gamma}=\left\{\right.$ diagonal matrix $\left.(\alpha, \alpha, \alpha), \alpha \in S^{1}\right\}$ and $\pi: U(3) \rightarrow P U(3)$ is its projection map. Let $\widetilde{T}$ and $\pi(\widetilde{T})=T$ be the maximal tori in $U(3)$ and $P U(3)$.

The fundamental class $\pi_{1}(\widetilde{T})$ is generated as $\left\langle\tilde{t}_{1}, \tilde{t}_{2}, \tilde{t}_{3}\right\rangle$ where

$$
\tilde{t}_{1}=\{\operatorname{diagonal}(\exp 2 \pi i t, 1,1), t \in[0,1]\}
$$

and so on. Then it is easily seen $\pi_{*}\left(\tilde{t}_{1}+\tilde{t}_{2}+\tilde{t}_{3}\right)=0$ and $\operatorname{Ker} \pi_{*}=\left\langle\tilde{t}_{1}+\tilde{t}_{2}+\tilde{t}_{3}\right\rangle$. Denote by $t_{j} \in H^{2}(B \widetilde{T} ; Z) \simeq H^{1}(\widetilde{T}) \simeq H_{1}(\widetilde{T})$ corresponding to $\tilde{t}_{j}$. Let us write $u, v \in H^{2}(B T ; Z)$ the corresponding elements to $\pi_{*}\left(\tilde{t}_{1}\right)$ and $\pi_{*}\left(\tilde{t}_{2}\right)$ respectively. 
Lemma 3.7. $\pi^{*} u=t_{1}-t_{3}$ and $\pi^{*} v=t_{2}-t_{3}$.

Proof. Since $H^{*}(B T ; Z) \rightarrow H^{*}(B \widetilde{T} ; Z)$ is monic we get the lemma from

$$
\left\langle\pi^{*} u, \tilde{t}_{i}\right\rangle=\left\langle u, \pi_{*} \tilde{t}_{i}\right\rangle=1 \quad(\text { resp. } 0,-1), \quad i=1 \quad(\text { resp. }=2,=3) .
$$

Here we note $\pi_{*}\left(\tilde{t}_{3}\right)$ corresponds to $-u-v$.

Let us write $T_{9}$ be the maximal torus of $U(9)$ and $\pi_{1}\left(T_{9}\right)=\left\langle t_{i j}\right| 1 \leq i, j \leq$ 3). Then $\tilde{\lambda}^{*}\left(t_{i j}\right)=t_{i}-t_{j}$. Since $\pi^{*}: H^{*}(B T ; Z) \rightarrow H^{*}(B \tilde{T} ; Z)$ is monic, $\lambda^{*}\left(t_{i j}\right)=-\lambda^{*}\left(t_{j i}\right)$ and $\lambda^{*}\left(t_{12}\right)=u-v, \lambda^{*}\left(t_{13}\right)=u, \lambda^{*}\left(t_{23}\right)=v$.

Lemma 3.8. The total Chern class in $H^{*}(B T)$ for $\lambda \mid T$ is

$$
\begin{aligned}
C(\lambda \mid T) & =\lambda^{*}\left(\pi\left(1+t_{i j}\right)\right)=\left(1-u^{2}\right)\left(1-v^{2}\right)\left(1-(u-v)^{2}\right) \\
& =1+(u+v)^{2}+(u+v)^{4}-u^{2} v^{2}\left(u^{2}+u v+v^{2}\right) .
\end{aligned}
$$

From Corollary 3.6,

\section{Lemma 3.9.}

$$
\begin{aligned}
C\left(\lambda \mid V_{1}\right) & =\pi\left(1+\lambda_{1} a+\lambda_{2} b\right) \\
& =\left(1-a^{2}\right)\left(1-b^{2}\right)\left(1-(a+b)^{2}\right)\left(1-(a-b)^{2}\right) \\
& =1-\left(a^{6}+a^{4} b^{2}+a^{2} b^{4}+b^{6}\right)+a^{2} b^{2}\left(a^{4}+a^{2} b^{2}+b^{4}\right)
\end{aligned}
$$

where $a, b \in H^{2}\left(B V_{1} ; Z / 3\right)$ is the Bockstein image of the dual element of $\tilde{a}$ and $\tilde{b}$, identifying $H^{1}\left(B V_{1} ; Z / 3\right) \simeq \operatorname{Hom}\left(V_{1} ; Z / 3\right)$.

The cohomology of $B P U(3)$ is given by Kono, Mimura, and Shimada [K-M-S].

Theorem 3.10. There is an algebra isomorphism

$$
H^{*}(B P U(3) ; Z / 3) \simeq Z / 3\left[y_{2}, y_{8}, y_{12}\right] \otimes \Lambda\left(y_{3}, y_{7}\right) / J
$$

where $\left|y_{i}\right|=i$ and $J$ is the ideal generated by $y_{2} y_{3}, y_{2} y_{7}$ and $y_{3} y_{7}+y_{2} y_{8}$. Moreover $y_{3}=\mathscr{B} y_{2}, y_{7}=\mathscr{P} y_{3}$, and $y_{8}=\mathscr{B} y_{7}$.

Note that $y_{2}^{2} y_{8}=-y_{3} y_{2} y_{7}=0$. Let $R_{1}=Z / 3\left[y_{2}, y_{12}\right]$ and $R_{2}=Z / 3\left[y_{8}, y_{12}\right]$. Then there are $Z / 3$-modules isomorphism

$$
H^{*}(B P U(3) ; Z / 3) \simeq y_{2}^{2} R_{1} \oplus Z / 3\left\{1, y_{2}, y_{3}, y_{7}\right\} R_{2} \text {. }
$$

Lemma 3.12. The ideal generated by $y_{2}^{2}$ is $y_{2}^{2} R_{1}$ in (3.11).

Consider the induced map $j_{1}: B V_{1} \rightarrow B P U(3)$. Let $a^{\prime}, b^{\prime}$ be the dual element of $\tilde{a}, \tilde{b}$ in $H^{1}\left(V_{1} ; Z / 3\right) \simeq \operatorname{Hom}\left(V_{1} ; Z / 3\right)$ and $\mathscr{B} a^{\prime}=a, \mathscr{B} b^{\prime}=b$. The commutative diagram

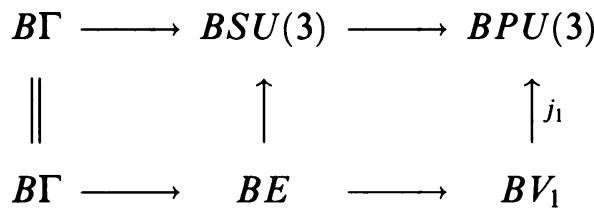

induces the map of spectral sequences

$$
E_{2}^{*, *}=H^{*}\left(B V_{1} ; H^{*}(B \Gamma ; Z / 3)\right) \stackrel{j_{1}^{*}}{\longleftarrow} \widetilde{E}_{2}^{*, *}=H^{*}\left(B P U(3) ; H^{*}(B \Gamma ; Z / 3)\right) \text {. }
$$

Since $H^{*}(B S U(3))$ and $H^{*}(B E)$ is known, we get $d_{2} c^{\prime}=a^{\prime} b^{\prime}$ in $E_{2}^{*, *}$ and $d_{2} c^{\prime}=y_{2}$ in $\widetilde{E}_{2}^{*, *}$. Therefore $j_{1}^{*} y_{2}=a^{\prime} b^{\prime}$. 
Lemma 3.14. $j_{1}^{*} y_{3}=\beta\left(a^{\prime} b^{\prime}\right)=a b^{\prime}-a^{\prime} b, j_{1}^{*} y_{7}=a^{3} b^{\prime}-a^{\prime} b^{3}$, and $j_{1}^{*} y_{8}=$ $a^{3} b-a b^{3}$.

The restriction $y_{i} \mid\langle a\rangle=0$ for $i \neq 12$, but $c_{6}(\lambda) \mid\langle b\rangle=b^{6} \neq 0$ from Lemma 3.9. Hence we can take $y_{12}=-c_{6}(\lambda)$.

Lemma 3.15. $\operatorname{Ker} j_{1}^{*}=y_{2}^{2} R_{1}$.

Proof. We need only prove $j_{1}^{*} \mid\left(y_{3} R_{1}+y_{7} R_{3}\right)$ is monic. From Lemmas 3.12 and 3.14, $\left(j_{1}^{*} y_{8}, j_{1}^{*} y_{12}\right)$ is a regular sequence in $Z / 3[a, b]$. Therefore $j_{1}^{*} f\left(y_{8}, y_{12}\right)$ $=0$ implies $f \equiv 0$. Let $j_{1}^{*}\left(y_{3} f+y_{7} g\right)=0$ and $j_{1}^{*} f=F, j_{1}^{*} g=G$. Taking modulo $a^{\prime}$, we get $a F+a^{3} G=0$ and taking modulo $b^{\prime}$, we have $b F+b^{3} G=0$. This implies $a b\left(a^{2}-b^{2}\right) G=0$, hence $G=0$. The regularity follows $g=0$. By the same argument, we also get $f=0$. Of course $j_{1}^{*} y_{2}^{2}=\left(a^{\prime} b^{\prime}\right)^{2}=0$.

Let $j_{2}: B T \rightarrow B P U(3)$ be the map from the inclusion of the maximal torus. Since $H^{*}(B T ; Z)$ is torsion free, and is generated by even dimensional elements, we have

$$
j_{2}^{*} y_{3}=j_{2}^{*} y_{7}=j_{2}^{*} y_{8}=0 .
$$

Since $j_{2}^{*} c_{2}(\lambda)=(u+v)^{2} \neq 0$, we can take $j_{2}^{*} y_{2}=u+v$. Therefore from Lemma 3.9,

$$
c_{1}(\lambda)=0, \quad c_{2}(\lambda)=\varepsilon y_{2}^{2}, \quad \varepsilon= \pm 1, \quad c_{3}(\lambda)=0 .
$$

The formula $c_{4}=\mathscr{P}^{2} c_{2}+c_{3} c_{1}-c_{2}\left(c_{2}+c_{1}^{2}\right)=\mathscr{P}^{2} c_{2}-c_{2}^{2}$ implies $y_{2}^{4}=(2 \varepsilon-1) y_{2}^{4}$. Hence we get $\varepsilon=1$.

Lemma 3.18. The Chern classes $c_{j}(\lambda)$ are represented by $y_{2}^{2}, y_{2}^{4}, y_{12}$, and $y_{8}^{2}$ for $j=2,4,6$, and 8 , respectively.

Comparing Lemmas 3.8 and 3.9 and considering the diagram

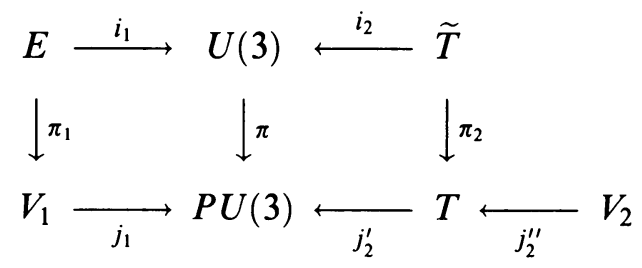

we have a short exact sequence

$$
0 \rightarrow \pi^{*}\left(\operatorname{Ker} j_{1}^{*}\right) \rightleftarrows H^{*}(B P U(3) ; Z / 3) \rightarrow \operatorname{Ker} \pi_{1}^{*} \rightarrow 0
$$

with $\pi^{*}\left(\operatorname{Ker} j_{1}^{*}\right)=Z / 3\left[c_{1}, c_{6}\right]\left\{c_{2}\right\} \subset H^{*}(B U(3) ; Z / 3)$ and $\operatorname{Ker} \pi_{1}^{*}=$ $Z / 3\left\{\mathscr{P}^{I} a^{\prime} b^{\prime}\right\} \subset H^{*}\left(V_{1} ; Z / 3\right)$.

Using Lemma 3.18, (3.19), and Lemma 3.14, we decide the Steenrod algebra structure of $H^{*}(B P U(3) ; Z / 3)$, and have proved Theorem 3.3.

Theorem 3.20. $\mathscr{P} 1 y_{3}=y_{7}, \mathscr{B} y_{7}=y_{8}, \mathscr{P}^{3} y_{7}=y_{7} y_{12}+y_{3} y_{8}^{2}, \mathscr{P}^{3} y_{8}=y_{8} y_{12}$, $\mathscr{P}^{1} y_{12}=y_{8}^{2}+y_{12} y_{2}^{2}, \mathscr{P}^{3} y_{12}=y_{12}\left(y_{2}^{6}-y_{12}\right)$.

\section{Brown-Peterson COHOMOLOGY OF BPU(3)}

Recall (3.11) in $\S 3$,

$$
A=H^{*}(B P U(3) ; Z / 3) \simeq y_{2}^{2} R_{1} \oplus Z / 3\left\{1, y_{2}, y_{3}, y_{7}\right\} R_{2}
$$


where $R_{1}=Z / 3\left[y_{2}, y_{12}\right], R_{2}=Z / 3\left[y_{8}, y_{12}\right]$ and $Q_{0} y_{2}=y_{3}, Q_{0} y_{7}=y_{8}$. Then $H\left(A ; Q_{0}\right) \simeq y_{2}^{2} R_{1} \oplus Z / 2\left[y_{12}\right]$ and its Poincare series is

$$
\text { P.S. }\left(H\left(A ; Q_{0}\right)\right)=\frac{t^{4}}{\left(1-t^{2}\right)\left(1-t^{12}\right)}+\frac{1}{\left(1-t^{12}\right)}=\frac{1}{\left(1-t^{4}\right)\left(1-t^{6}\right)},
$$

which is the Poincare series of the polynomial algebra of degree 4 and 6 and is equal to the Poincare series of $H^{*}\left(B P U(3) ; Q_{0}\right)$. Therefore the Bockstein spectral sequence collapses, i.e., $E_{1} \simeq E_{\infty}$. This means $H^{*}(B P U(3))$ has no higher 3-torsion.

Consider the Atiyah-Hirzebruch spectral sequence

$$
E_{2}^{*, *}=H^{*}\left(B P U(3) ; B P^{*}\right) \Rightarrow B P^{*}(B P U(3)) \text {. }
$$

The $E_{2}$-term is given by

$$
E_{2}^{*, *}=B P^{*} \otimes\left\{y_{2}^{2} \widetilde{R}_{1} \oplus \widetilde{R}_{3} \oplus R_{2} y_{3} \oplus R_{2} y_{8}\right\}
$$

where $\widetilde{R}_{1}=Z_{(3)}\left[y_{2}, y_{12}\right], \widetilde{R}_{3}=Z_{(3)}\left[y_{12}\right]$. The first nonzero differential is $d_{2 p-1}=v_{1} \otimes Q_{1}$. Since $Q_{1} y_{3}=y_{8}$, we get

$$
E_{2 p}^{*, *} \simeq B P^{*}\left\{y_{2}^{2} \widetilde{R}_{1} \oplus \widetilde{R}_{3}\right\} \oplus B P^{*} /\left(3, v_{1}\right) \otimes\left\{R_{2} y_{8}\right\} .
$$

These are all even dimensional elements and $E_{2 p}^{*, *} \simeq E_{\infty}^{*, *}$.

Therefore we see $B P^{*}(B P U(3))=B P^{\text {even }}(B P U(3))$. Moreover each element in $E_{2 p}^{* * *}$ is nonnilpotent, we get $B P^{*}(B P U(3))$ has no nonzero nilpotent element. Hence (1)-(3) and (7) in the introduction hold.

However (6) does not hold as following. Recall the representation ring $R(S U(3))=Z[l, \bar{l}]$ where $l$ is represented by the character of the canonical representation $\rho$ and $\bar{l}$ is its conjugation. The representation ring of $P S U(3)$ is easily deduced as the subring generated by

$$
\left\{l^{c} \bar{l}^{d} \mid c+2 d \equiv 0 \bmod 3, c, d \in \mathbb{Z}\right\} .
$$

For $c, d \geq 0$, let $M(c, d)$ is the corresponding $P S U(3)$-module.

Lemma 4.5.

$$
\chi_{M(c, d)}\left(\tilde{a}^{i} \tilde{b}^{j}\right)= \begin{cases}3^{c+d}, & i \equiv j \equiv 0 \bmod 3, \\ 0, & \text { otherwise. }\end{cases}
$$

Proof. This is easily seen from the facts $T_{r}(f \times g)=T_{r}(f) T_{r}(g)$ and

$$
\chi_{i}\left(\bar{a}^{j}\right)=\chi_{i}\left(\bar{b}^{j}\right)= \begin{cases}3, & j \equiv 0 \bmod 3, \\ 0, & \text { otherwise. }\end{cases}
$$

Corollary 4.6. $M(c, d) \mid V_{1}=3^{c+d-2}$ (regular representation).

From Lemma 3.9, $y_{8} \mid V_{1} \notin$ Image $\lambda^{*}\left(H^{*}(B U(3)) \rightarrow H^{*}\left(B V_{1}\right)\right)$ where $\lambda$ is the regular representation.

Corollary 4.7. Since $y_{8} \notin \mathrm{Ch}_{B P}(B P U(3))$ and $y_{2}^{3} \notin \mathrm{Ch}_{B P}(B P U(3))$, we have

$$
\mathrm{Ch}_{B P}(B P U(3)) \neq B P^{*}(B P U(3)) \text {. }
$$

We now consider the $B P^{*}$-module structure of $B P^{*}(B P U(3))$. The $B P^{*}$ algebra structure of $B Z / p$ is well known:

$$
B P^{*}[[u]] /[p](u), \quad|u|=2,
$$


where $[p](u)=u+_{B P} \cdots+_{B P} u$ is the $p$ th product of the formal group law over $B P^{*}$-theory. Note that $[p](u)=\sum v_{n} u^{p^{n}} \bmod \left(p, v_{1}, \ldots\right)^{2}$ and $Q_{n}(\alpha)=$ $\rho(u)^{p^{n}}$ where $H^{*}(B Z / p ; Z / p) \simeq Z / p[\rho(u)] \otimes \Lambda(\alpha)$ and $\rho: B P \rightarrow Z / p$ be the natural map. This fact extends as the following lemma.

Lemma 4.8 [Y1]. If there is a relation $\sum v_{n} x_{n}=0$ in $B P^{*}(X)$, then there exists $y \in H^{*}(X ; Z / p)$ such that $Q_{i}(y)=\rho\left(x_{i}\right)$.

Theorem 4.9. Let us fix elements $\tilde{f}\left(y_{i}\right) \in B P^{*}(B P U(3))$ with $\rho(\tilde{f})=f$, i.e., $\rho\left(\tilde{y}_{12}\right)=y_{12}, \rho\left(\tilde{y}_{2}^{2}\right)=y_{2}^{2}$. There is a $B P^{*}$-module isomorphism $B P^{*}(B P U(3))$ $\simeq\left(B P^{*} S_{2} \tilde{y}_{2}^{2} \oplus B P^{*}\{1\} \oplus B P^{*} S_{8} \tilde{y}_{8}\right) \otimes S_{12} /\left(I_{1}, I_{2}\right)$ where $S_{i}=Z_{(3)}\left[\left[\tilde{y}_{i}\right]\right]$ and

$$
I_{1} \equiv \sum v_{n} \widetilde{Q}_{n}\left(y_{7}\right), \quad I_{2} \equiv \sum v_{n} \widetilde{Q}_{n}\left(y_{3}\right) \quad \bmod \left(3, v_{1}, \ldots\right)^{2} .
$$

Proof. From (4.3), there are relations such that $I_{1}=p \tilde{y}_{8}+\cdots$ and $I_{2}=v_{1} \tilde{y}_{8}+$ $\ldots$. Since $\operatorname{Ker} \rho\left(B P^{*}(B P U(3)) \rightarrow H^{*}(B P U(3) ; Z / 3)\right)=\left(v_{1}, v_{2}, \ldots\right)$, we have the theorem from Lemma 4.8 and $Q_{0} y_{7}=y_{8}, Q_{1} y_{3}=y_{8}$.

Lemma 4.10. Let us write $e_{i}=Q_{i} y_{3}$ and $X=y_{8}^{3}, Y=y_{12}^{2}$. For $i=2 j+1>0$, $e_{i}=f_{i}(X, Y) y_{8}$ and $e_{i+1}=g_{i+1}(X, Y) y_{8} y_{12}$. Moreover,

$$
g_{i+1}=\left(f_{i}^{\prime}\right)^{3} Y X^{2}+f_{i}^{3}, \quad f_{i+2}=\left(g_{i+1}^{\prime}\right)^{3} Y^{3} X+g_{i+1}^{3}\left(Y^{2}+X^{2}\right),
$$

where $f^{\prime}=\partial f / \partial Y$ and $g^{\prime}=\partial g / \partial Y$. In particular, $e_{i}=0, y_{8}, y_{12} y_{8}$, $y_{8}\left(X^{2}+Y^{2}\right)$ for $i=0,1,2,3$, respectively.

Proof. Let us denote $\mathscr{P}^{\#} a$ by \#=1/2|a|. For $i \geq 1$,

$$
e_{i+1}=Q_{i+1} y_{3}=\left(\mathscr{P}^{p^{i}} Q_{i}-Q_{i} \mathscr{P}^{p^{i}}\right) y_{3}=\mathscr{P}^{p^{i}} Q_{i} y_{3}=\mathscr{P}^{p^{i}} e_{i} .
$$

Here we note $\left|e_{i}\right|=2\left(p^{i}-1\right)$. Therefore

$$
\begin{aligned}
e_{i+1} & =\mathscr{P}^{\#-1} e_{i}=\mathscr{P}^{\#-1} f \mathscr{P}^{\#} y_{8}+\mathscr{P}^{\#} f \mathscr{P}^{\#-1} y_{8} \\
& =\left(\mathscr{P}^{\#-1} f\right) y_{8}^{3}+f^{3} y_{8} y_{12} .
\end{aligned}
$$

Note $\mathscr{P}^{\#-1} X=0$ and $\mathscr{P}^{\#-1} Y=2 y_{12}^{3}\left(-y_{8}^{4}\right)=y_{12}^{3} y_{8}^{4}$ since $\mathscr{P}^{5} y_{12}=-y_{8}^{4}$. Therefore

$$
\mathscr{P}^{\#-1} \sum \lambda_{i j} X^{i} Y^{j}=\sum j \lambda_{i j}\left(X^{i}\right)^{3}\left(Y^{j-1}\right)^{3} y_{12}^{3} y_{8}^{4} \text {. }
$$

This means $\mathscr{P}^{\#-1} f=\left(f^{\prime}\right)^{3} Y X y_{12} y_{8}$ and $e_{i+1}=\left\{\left(f^{\prime}\right)^{3} Y X^{2}+f^{3}\right\} y_{8} y_{12}$.

Next consider $e_{i+2}$. We have

$$
\begin{aligned}
e_{i+2} & =\mathscr{P}^{i+1} e_{i}=\mathscr{P}^{\#-1}\left(g y_{8} y_{12}\right) \\
& =\left(\left(g^{\prime}\right)^{3} Y X y_{12} y_{8}\right) y_{12}^{3} y_{8}^{3}+g^{3}\left(y_{8} y_{12}^{4}+y_{8}^{7}\right) \\
& =y_{8}\left(\left(g^{\prime}\right)^{3} Y^{3} X+g^{3}\left(Y^{2}+X^{2}\right)\right) .
\end{aligned}
$$

Lemma 4.11. Let us write $d_{i}=Q_{i} y_{7}$. Then for $i \geq 2, d_{i}=\left(e_{i-1}\right)^{3}$. In particular, $d_{i}=y_{8}, 0, y_{8}^{3},\left(y_{12} y_{8}\right)^{3}$ for $i=0,1,2,3$.

Proof. By induction, for $i \geq 2$,

$$
Q_{i} d_{i}=\mathscr{P}^{p} d_{i-1}=\mathscr{P}^{p}\left(e_{i-2}\right)^{p}=\left(\mathscr{P}^{\#-1} e_{i-2}\right)^{3} .
$$

Lemma 4.12. A greatest common divisor of $\left(e_{i} / y_{8}\right)$ for all $i \geq m$ is equal to 1 . Proof. We assume that $f_{i}$ has no double root and $X, Y$ as root. Then $f_{i}$ and $f_{i}^{\prime}$ have no common divisor. Suppose $g_{i+1}$ and $f_{i}$ have same root. Then 
from Lemma 4.10, $f_{i}$ and $f_{i}^{\prime}$ have same root and this contradicts to the first assumption. Since $g_{i+1}^{\prime}=\left(f_{i}\right)^{3} X^{2}, g_{i+1}$ and $g_{i+1}^{\prime}$ do not have the same divisor since so do not $f_{i}$ and $f_{i}^{\prime}$. Similar facts hold for $i+2$.

Corollary 4.13. The elements $I_{1}^{\prime}=I_{1} / y_{8}, I_{2}^{\prime}=I_{2} / y_{8}^{3}$ are prime for each $m \geq 0$, that is, for $m \geq 0$ if $a I_{1}^{\prime}+b I_{2}^{\prime}=0$ in $P(m)^{*} \otimes S_{8} \otimes S_{12}$, then $a=a^{\prime} I_{2}^{\prime}$ and $b=-a^{\prime} I_{1}^{\prime}$ in $P(m)^{*} \otimes S_{8} \otimes S_{12}$.

Proof. Note that $I_{1}^{\prime}=v_{m} f_{m}+v_{m+1} g_{m} y_{12}+\cdots$ and $I_{2}^{\prime}=v_{m}\left(g_{m-1} y_{12}\right)^{3}+$ $v_{m+1}\left(f_{m}\right)^{3}+\cdots$. If $I_{1}^{\prime}=a b$, then $a$ is unit or $b$ is unit from Lemma 4.12. These facts follow the corollary.

Theorem 4.14. $P(m)^{*}(B P U(3)) \simeq P(m)^{*} \otimes_{B P *} B P^{*}(B P U(3))$.

Proof. Suppose that $p x=0$ in $B P^{*}(B P U(3))$. Then $p \tilde{x}=a I_{1}+b I_{2}$ in $B P^{*} \otimes S_{8} \otimes S_{12} \tilde{y}_{8}^{2}$. Hence $0=a I_{1}+b I_{2}$ in $P(1)^{*} \otimes S_{8} \otimes S_{12} \tilde{y}_{8}^{2}$. This means also $0=a I_{1}^{\prime}+b I_{2}^{\prime}$. Hence $a=I_{2}^{\prime} a^{\prime}$ and $b=-I_{1}^{\prime} a^{\prime} \bmod p$. Therefore $a=I_{2} a^{\prime}+p a^{\prime \prime}, b=-I_{1} a^{\prime}+p b^{\prime \prime}$ in $B P^{*} \otimes S_{8} \otimes S_{12}$. Hence $p \tilde{x}=p I_{1} a^{\prime \prime}+p I_{2} b^{\prime \prime}$. This means $\tilde{x}=I_{1} a^{\prime \prime}+I_{2} b^{\prime \prime}$ and $\tilde{x}=0$ in $B P^{*}(B P U(3))$. Therefore there is no $p$-torsion in $B P^{*}(B P U(3))$. Hence when $m=1$, the theorem is proved. The case $m \geq 2$ are also proved by the same argument from Corollary 4.13.

Therefore $G=P U(3)$ satisfies (1)-(7) in the introduction.

$$
\text { 5. } S O(4) \text { FOR } p=2 \text { AND } F_{4} \text { FOR } p=3
$$

Recall $H^{*}(B S O(4) ; Z / 2)=Z / 2\left[w_{2}, w_{3}, w_{4}\right]$ and $Q_{0} w_{2}=w_{3}$. It is known that there is no higher 2-torsion

$$
H^{*}(B S O(4))_{(2)} \simeq\left(Z_{(2)}\left[w_{2}^{2}\right] \oplus Z / 2\left[w_{3}, w_{2}^{2}\right]\left\{w_{3}\right\}\right) \otimes Z_{(2)}\left[w_{4}\right] .
$$

Consider the Atiyah-Hirzebruch spectral sequence

$$
E_{2}^{*, *}=H^{*}\left(B S O(4) ; B P^{*}\right) \Rightarrow B P^{*}(B S O(4)) \text {. }
$$

From (2.6), $Q_{1} w_{3}=w_{3}^{2}$ and $Q_{1} w_{4}=w_{4} w_{3}$. Let us write $A=B P^{*}\left[w_{2}^{2}, w_{4}^{2}\right]$. Then from $d_{2 p-1}=v_{1} \otimes Q_{1}$, we have

$$
E_{2 p}^{*, *}=A \oplus A /\left(2, v_{1}\right)\left[w_{3}^{2}\right]\left\{w_{3}^{2}, w_{4} w_{3}\right\}
$$

This module is a direct product of a free $B P^{*}$-module and a free $B P^{*} /\left(2, v_{1}\right)$ module. Hence the next nonzero differential is $d_{2 p^{2}-1}=v_{2} \otimes Q_{2}$. Since $Q_{2} w_{4} w_{3}=w_{3}^{2} w_{4}^{2}$, we get

$$
E_{2 p^{2}}^{* *}=A\left\{1,2 w_{4}\right\} \oplus B\left\{w_{3}^{2}\right\} /\left(2, v_{1}\right) \oplus B\left[w_{4}^{2}\right]\left\{w_{3}^{2} w_{4}^{2}\right\} /\left(2, v_{1}, v_{2}\right)
$$

where $B=B P^{*}\left[w_{3}^{2}, w_{2}^{2}\right]$. Since $E_{2 p^{2}}^{*, *}$ is generated by even dimensional elements, we see $E_{2 p^{2}} \simeq E_{\infty}$.

Theorem 5.5. There is a $B P^{*}$-module isomorphism

$$
\begin{aligned}
B P^{*}(B S O(4)) \simeq & \widetilde{A}\left\{1,2 \tilde{w}_{4}\right\} \oplus \widetilde{B}\left\{\tilde{w}_{3}^{2}\right\} /\left(I_{1}, I_{2}\right) \\
& \oplus \widetilde{B}\left[\left[\tilde{w}_{4}^{2}\right]\right]\left\{\tilde{w}_{3}^{2} \tilde{w}_{4}^{2}\right\} /\left(I_{1} \tilde{w}_{4}^{2}, I_{2} \tilde{w}_{4}^{2}, I_{3}\right)
\end{aligned}
$$

where $\tilde{A}=B P^{*}\left[\left[\tilde{w}_{2}^{2}, \tilde{w}_{4}^{2}\right]\right], \widetilde{B}=B P^{*}\left[\left[\tilde{w}_{2}^{2}, \tilde{w}_{3}^{2}\right]\right]$, and $I_{1} \equiv \sum v_{n} \widetilde{Q}_{n}\left(w_{2} w_{3}\right)$, $I_{2} \equiv \sum v_{n} \widetilde{Q}_{n}\left(w_{3}\right)$, and $I_{3} \equiv \sum v_{n} \widetilde{Q}_{n}\left(w_{3} w_{4}\right) \bmod \left(2, v_{1}, \ldots\right)^{2}$.

Properties (1)-(3) and (7) hold immediately. Properties (4), (5) are proved by the arguments similar to the case $G=P U(3)$, but a little difficult. 
Lemma 5.6. Let $I_{1}^{\prime}=I_{1} /\left(\tilde{w}_{3}^{2}\right), I_{2}^{\prime}=I_{2} /\left(\tilde{w}_{3}^{2}\right)$, and $I_{3}^{\prime}=I_{3} /\left(w_{3}^{2} w_{4}^{2}\right)$. The ideals $I_{1}^{\prime}$ and $I_{2}^{\prime}$ are prime in $P(m)^{*} \otimes_{B P^{*}} B$. If $a I_{3}^{\prime} \in \operatorname{Ideal}\left(I_{2}, I_{1}\right)$ in $P(m)^{*} \otimes_{B P^{*}}$ $B\left[\left[w_{4}^{2}\right]\right]$, then $a \in \overline{\operatorname{Ideal}}\left(I_{2}, I_{1}\right)$.

Outline of proof. By the arguments similar to Lemma 4.10, we have $n \geq 2$

$$
\begin{aligned}
Q_{n} w_{3} w_{2} & =\left(w_{3} f(X, Y)\right)^{4} \quad \text { where } X=w_{2}^{3}, Y=w_{3}^{2}, \\
Q_{n+1} w_{3} w_{2} & =\left(w_{3} w_{2}\left(f^{2}+Y X\left(f^{\prime}\right)^{2}\right)\right)^{4} \quad \text { where } f^{\prime}=\partial f / \partial X,
\end{aligned}
$$

and $Q_{n} w_{3}=2 \sqrt{Q_{n+1}\left(w_{3} w_{2}\right)}$. Hence we can prove $I_{1}^{\prime}, I_{2}^{\prime}$ are prime in $\widetilde{B} \otimes_{B P^{*}}$ $P(m)^{*}$. Moreover we can see for $n \geq 2$

$$
Q_{n}\left(w_{3} w_{4}\right)=w_{2}^{2} w_{4}^{2^{n-2}} \quad \bmod w_{2}^{4}
$$

Suppose $a I_{3}^{\prime}=b_{1} I_{1}^{\prime}+b_{2} I_{2}^{\prime}$ in $P(m)^{*} \otimes_{B P^{*}} \widetilde{B}\left[\left[\tilde{w}_{4}^{2}\right]\right]$. Here recall that (we assume $m$ even)

$$
\begin{aligned}
& I_{1}^{\prime}=\left(v_{m}+v_{m+2} \tilde{w}_{3}^{*}+v_{m+4} \tilde{w}_{3}^{*}+\cdots\right) \tilde{w}_{3}^{*} \bmod \tilde{w}_{2}^{2}, \\
& I_{2}^{\prime}=\left(v_{m+1}+v_{m+3} \tilde{w}_{3}^{*}+\cdots\right) \tilde{w}_{3}^{*} \bmod \tilde{w}_{2}^{2}, \\
& I_{3}^{\prime}=\left(v_{m}+v_{m+1} \tilde{w}_{4}^{*}+v_{m+2} \tilde{w}_{4}^{*}+\cdots\right) \tilde{w}_{4}^{*} \bmod \tilde{w}_{2}^{2} .
\end{aligned}
$$

Then we can easily see $a=b_{1}^{\prime} I_{1}^{\prime}+b_{2}^{\prime} I_{2}^{\prime} \bmod \tilde{w}_{2}^{2}$. Now take out $\left(\tilde{a}=a-b_{1}^{\prime} I_{1}^{\prime}-b_{2}^{\prime} I_{2}^{\prime}\right) I_{3}^{\prime}$ from both sides of the supposition. Next, divide both sides by $\tilde{w}_{2}^{2}$. Using these arguments, we can prove this lemma.

From Lemma 5.6, we can prove

$$
P(m)^{*}(B S O(4)) \simeq P(m)^{*} \otimes_{B P^{*}} B P^{*}(B S O(4))
$$

and also prove $(4),(5)$ in the introduction.

Remark 5.8. It is easily seen

$$
\begin{aligned}
& B P^{*}(B S O(3)) \simeq B P^{*}(B S O(4)) /\left(\tilde{w}_{4}^{2}, 2 \tilde{w}_{4}\right)
\end{aligned}
$$

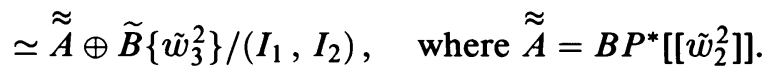

Next consider $G=F_{4}, p=3$. By Toda [T1] cohomology of $H^{*}\left(B F_{4} ; Z / 3\right)$ is known.

Theorem 5.9 (Toda).

$$
H^{*}\left(B F_{4} ; Z / 3\right) \simeq Z / 3\left[x_{36}, x_{48}\right] \otimes C
$$

for

$$
C=Z / 3\left[x_{4}, x_{8}\right] \otimes\left\{1, x_{20}, x_{20}^{2}\right\} \oplus Z / 3\left[x_{26}\right] \otimes \Lambda\left(x_{9}\right) \otimes\left\{1, x_{20}, x_{21}, x_{25}\right\}
$$

where two terms of $C$ have the intersection $\left\{1, x_{20}\right\}$.

Toda also determined the Steenrod algebra structure completely. (See Theorems I-III in [T1]). For example, $\beta x_{i}=x_{i+1}$ if $x_{i+1}$ exists. Let $R_{1}=$ $Z / 3\left[x_{4}, x_{8}\right], R_{2}=Z / 3\left[x_{8}\right]$, and $R_{3}=Z / 3\left[x_{26}\right]$. Then it is easily computed in $C$

$$
\begin{aligned}
\operatorname{Ker} Q_{0}= & \{1\} \oplus x_{4} R_{1} \oplus x_{8}^{2} R_{2} \oplus x_{20} x_{4} R_{1} \oplus x_{20} x_{8} R_{2} \\
& \oplus x_{20}^{2} R_{1} \oplus x_{26} R_{3} \oplus x_{21} R_{3} \oplus x_{9} R_{3} \oplus x_{9} x_{21} R_{3}
\end{aligned}
$$


and

$\operatorname{Ker} Q_{0} / \operatorname{Im} Q_{0} \simeq\{1\} \oplus x_{4} R_{1} \oplus x_{8}^{2} R_{2} \oplus x_{4} x_{20} R_{1} \oplus x_{8} x_{20} R_{2} \oplus x_{20}^{2} R_{1}$.

The Poincare series of $\operatorname{Ker} Q_{0} / \operatorname{Im} Q_{0}$ is

$$
1+\frac{t^{4}+t^{24}+t^{40}}{\left(1-t^{4}\right)\left(1-t^{8}\right)}+\frac{t^{16}+t^{28}}{1-t^{8}}=\frac{1}{\left(1-t^{4}\right)\left(1-t^{16}\right)\left(1-t^{12}\right)\left(1-t^{24}\right)} .
$$

Therefore we see

Proposition 5.10. There is no higher torsion in $H^{*}\left(B F_{4}\right)_{(3)}$.

Remark. This fact is also easily proved by using the Becker-Gottlieb transfer. For the fibering $\pi \rightarrow B \operatorname{Spin}(9) \stackrel{p}{\longrightarrow} B F_{4}$, we have $p_{*} p^{*}=\times \chi(\pi)=\times 3$. Since $H^{*}(B \operatorname{Spin}(9))_{(3)}$ is 3-torsion free, there is no higher 3-torsion. This argument is also applied for $P U(3), p=3$ and $E_{8}, p=5$.

Consider the Atiyah-Hirzebruch type spectral sequence $E_{2}^{*, *}=H^{*}\left(B F_{4} ; B P^{*}\right)$ $\Rightarrow B P^{*}\left(B F_{4}\right)$. Let $S_{1}=B P^{*}\left[x_{4}, x_{8}\right], S_{2}=B P^{*}\left[x_{8}\right], S_{3}=B P^{*} /(3)\left[x_{26}\right]$, and $D=Z_{(3)}\left[x_{36}, x_{48}\right]$. Then

$$
\begin{aligned}
E_{2}^{*, *}=\left(B P^{*}\{1\} \oplus S_{1} x_{4} \oplus S_{2} x_{8}^{2} \oplus S_{1} x_{4} x_{20} \oplus S_{2} x_{8} x_{20} \oplus S_{1} x_{20}^{2}\right. \\
\\
\left.\oplus S_{3} \otimes\left\{x_{26}, x_{21}, x_{9}, x_{9} x_{21}\right\}\right) \otimes D .
\end{aligned}
$$

The first nonzero differential is $d_{2 p-1}=v_{1} \otimes Q_{1}$ and we know, from Toda, $Q_{1} x_{4}=x_{9}, Q_{1} x_{20}=x_{25}, Q_{1} x_{21}=x_{26}$. Let

$$
A=\left(B P^{*}\left\{1,3 x_{4}\right\} \oplus S_{1} x_{4}^{2} \oplus S_{2} x_{8}^{2} \oplus S_{1} x_{4} x_{20} \oplus S_{2} x_{8} x_{20} \oplus S_{1} x_{20}^{2}\right) .
$$

Then

$$
E_{2 p}^{* * *}=\left(A \oplus S_{3} /\left(v_{1}\right)\left\{x_{9}, x_{26}\right\}\right) \otimes D .
$$

Next nonzero differential is $d_{2 p^{2}-1}=v_{2} \otimes Q_{2}$ and $Q_{2} x_{9}=x_{26}$. Therefore

$$
E_{2 p^{2}}^{*, *}=\left(A \oplus S_{3} /\left(v_{1}, v_{2}\right)\left\{x_{26}\right\}\right) \otimes D .
$$

Since this is generated by even dimensional elements $E_{2 p^{2}}^{*, *} \simeq E_{\infty}^{*, *}$. The properties (1)-(3), (7) hold from (5.13).

Theorem 5.14. There is a $B P^{*}$-module isomorphism

$$
B P^{*}\left(B F_{4}\right) \simeq \widetilde{A} \otimes \widetilde{D} \oplus B P^{*}\left[\left[\tilde{x}_{26}\right]\right]\left\{\tilde{x}_{26}\right\} \otimes \widetilde{D} /\left(I_{1}, I_{2}, I_{3}\right)
$$

where $I_{1} \equiv \sum v_{n} \widetilde{Q}_{n}\left(x_{25}\right), I_{2} \equiv \sum v_{n} \widetilde{Q}_{n}\left(x_{21}\right)$, and

$$
I_{3} \equiv \sum v_{n} \widetilde{Q}_{n}\left(x_{9}\right) \bmod \left(3, v_{1}, \ldots\right)^{2} .
$$

The properties (4) and (5) are proved by the arguments similar to the arguments 4.11-4.14 and 5.6, but some more complicated. Note that $Q_{n}\left(x_{i}\right)$ are computed by Theorem III in [T]. For example, $Q_{n}\left(x_{9}\right)=0,0, x_{26}, x_{36} x_{26}$, $x_{26}\left(x_{36}^{4}+x_{48}^{3}\right)$ for $n=0,1,2,3,4$, respectively. For $n=$ even $\geq 2, Q_{n}\left(x_{9}\right)=$ $x_{26} f(X, Y)$ with $X=x_{36}^{4}, Y=x_{48}^{3}$. Then

$$
Q_{n+1}\left(x_{9}\right)=x_{36} x_{26}\left(\left(f^{\prime}\right)^{3} X^{2} Y+f^{3}\right)=x_{36} x_{26} g \bmod x_{26}^{2}
$$

and

$$
Q_{n+2}\left(x_{9}\right)=x_{26}\left(Y X^{3}\left(g^{\prime}\right)^{3}+g^{3}(X+Y)\right) \bmod x_{26}^{2},
$$


where $f^{\prime}=\partial f / \partial X$.

6. $G=G_{2}, F_{4}, E_{6}$, AND $\operatorname{Spin}(n), n \leq 10$

The mod 2 cohomology of $B \operatorname{Spin}(n)$ is given by Quillen [Q2] (6.1)

$$
H^{*}(B \operatorname{Spin}(n) ; Z / 2) \simeq Z / 2\left[w_{2^{h}}(\Delta)\right] \otimes Z / 2\left[w_{2}, \ldots, w_{n}\right] /\left(Q_{i} w_{2} \mid 0 \leq i \leq h\right)
$$

where $\Delta$ is a spin representation of $\operatorname{Spin}(n)$ and $2^{h}$ is the Radon-Hurwitz number (see [Q2, p. 210]). When $n \leq 9, H^{*}(B \operatorname{Spin}(n) ; Z / 2)$ is a polynomial algebra generated by $w_{4}, w_{6}, w_{7}, w_{8}$, and $w_{2^{h}}(\Delta)$. Note that $G_{2} \hookrightarrow \operatorname{Spin}(7)$ and

$$
H^{*}\left(B G_{2} ; Z / 2\right)=Z / 2\left[w_{4}, w_{6}, w_{7}\right]
$$

Cohomology $B G_{2}$ and $B F_{4}$ is given by Borel [B].

At first we study $B P^{*}\left(B G_{2}\right)$ and consider the Atiyah-Hirzebruch spectral sequence. Since $Q_{0} w_{6}=w_{7}$, we have

$$
E_{2}^{*, *}=A \oplus A /(2)\left[w_{7}\right]\left\{w_{7}\right\} \quad \text { where } A=B P^{*}\left[w_{4}, w_{6}^{2}\right]
$$

Since $Q_{1} w_{4}=w_{7}$, we get for $B=B P^{*}\left[w_{4}^{2}, w_{6}^{2}\right]$

$$
E_{2 p}^{*, *}=B \oplus B\left\{2 w_{4}\right\} \oplus B /\left(2, v_{1}\right)\left[w_{7}\right]\left\{w_{7}\right\} .
$$

The facts $Q_{2} w_{7}=w_{7}^{2}$ and $d_{2 p^{2}-1}\left(2 w_{4}\right) \neq v_{2} w_{4} w_{7}$ because $d_{2 p^{2}-1}\left(v_{2} w_{4} w_{7}\right)=$ $v_{2} w_{7}^{2} \neq 0$, imply

$$
E_{2 p^{2}}^{*, *}=B \oplus B\left\{2 w_{4}\right\} \oplus B /\left(2, v_{1}, v_{2}\right)\left[w_{7}^{2}\right]\left\{w_{7}^{2}\right\}
$$

Theorem 6.6. $E_{\infty}^{*, *} \simeq E_{2 p^{2}}^{*, *}$ and we get

$$
B P^{*}\left(B G_{2}\right) \simeq \widetilde{B} \oplus \widetilde{B}\left\{2 \tilde{w}_{4}\right\} \oplus \widetilde{B}\left[\left[\tilde{w}_{7}^{2}\right]\right]\left\{\tilde{w}_{7}^{2}\right\} /\left(I_{1}, I_{2}, I_{3}\right)
$$

where $I_{1}=\sum v_{n} \widetilde{Q}_{n}\left(w_{7} w_{6}\right), \quad I_{2}=\sum v_{n} \widetilde{Q}_{n}\left(w_{7} w_{4}\right), \quad I_{3}=\sum v_{n} \widetilde{Q}_{n}\left(w_{7}\right)$, and $\widetilde{B}=B P^{*}\left[\left[\tilde{w}_{4}^{2}, \tilde{w}_{6}^{2}\right]\right]$. Hence $B P^{*}\left(B G_{2}\right)$ satisfies $(1)-(3),(7)$.

Remark 6.7. The ideal $\left(I_{1}, I_{2}, I_{3}\right)$ seems to satisfy the similar property in Lemma 5.6. However we cannot prove it yet.

Let us write by $E_{r}^{*, *}(B G)$ the $E_{r}$-term of the Atiyah-Hirzebruch type spectral sequence converging to $B P^{*}(B G)$.

Now we consider $B \operatorname{Spin}(n), \quad n=7,8,9$, while $H^{*}(B \operatorname{Spin}(n) ; Z / 2)$ for $n \leq 6$ is generated by even dimensional elements. The cohomology

$$
H^{*}(B \operatorname{Spin}(7) ; Z / 2) \simeq H^{*}\left(B G_{2} ; Z / 2\right) \otimes Z / 2\left[w_{8}\right]
$$

and $Q_{i} w_{8}=0$ for $0 \leq i \leq 1$ and $Q_{2} w_{8}=w_{8} w_{7}$. Therefore

$$
E_{r}^{*, *}(B \operatorname{Spin}(7)) \simeq E_{r}^{*, *}\left(B G_{2}\right) \otimes Z_{(2)}\left[w_{8}\right] \text { for } r \leq 2 p^{2}-2,
$$

and we get

$$
\begin{aligned}
E_{2 p^{2}}(B \operatorname{Spin}(7)) \simeq & \left(E_{2 p^{2}}\left(B G_{2}\right) \oplus B /\left(2, v_{1}\right)\left\{w_{8}\right\} \oplus B\left\{2 w_{4} w_{8}\right\}\right. \\
& \left.\oplus B /\left(2, v_{1}, v_{2}\right)\left[w_{7}^{2}\right]\left\{w_{8} w_{7}\right\}\right)\left[w_{8}^{2}\right]
\end{aligned}
$$

Since $Q_{3} w_{8} w_{7}=w_{8}^{2} w_{7}^{2}$, we have

$$
\begin{aligned}
E_{2 p^{3}}(B \operatorname{Spin}(7)) \simeq\left(E_{2 p^{2}}\left(B G_{2}\right) \oplus B /\left(2, v_{1}\right)\left\{w_{8}\right\} \oplus B\left\{2 w_{2} w_{8}\right\}\right. \\
\left.\oplus B /\left(2, v_{1}, v_{2}, v_{3}\right)\left[w_{7}^{2}\right]\left\{w_{8}^{2} w_{7}^{2}\right\}\right)\left[w_{8}^{2}\right] .
\end{aligned}
$$


Therefore $E_{2 p^{3}} \simeq E_{\infty}$ and the properties (1)-(3), (7) hold for $G=\operatorname{Spin}(7)$.

The cohomologies are

$$
H^{*}(B \operatorname{Spin}(8) ; Z / 2) \simeq H^{*}(B \operatorname{Spin}(7) ; Z / 2) \otimes Z / 2\left[w_{8}^{\prime}\right],
$$

and

$$
H^{*}(B \operatorname{Spin}(9) ; Z / 2) \simeq H^{*}(B \operatorname{Spin}(7) ; Z / 2) \otimes Z / 2\left[w_{16}\right] .
$$

We can compute

$E_{2 p^{4}}(B \operatorname{Spin}(8))$

$$
\begin{aligned}
\simeq\left(E_{2 p^{3}}(B \operatorname{Spin}(7)) \oplus E_{2 p^{3}}(B \operatorname{Spin}(7))^{\prime} \ominus E_{2 p^{3}}\left(B G_{2}\right)\right. \\
\oplus\left(B\left(2, v_{1}, v_{2}\right)\left\{w_{8} w_{8}^{\prime}\right\} \oplus B\left\{2 w_{4} w_{8} w_{8}^{\prime}\right\}\right. \\
\left.\left.\oplus B /\left(2, v_{1}, v_{2}, v_{3}, v_{4}\right)\left[w_{7}^{2}\right]\left\{\left(w_{8}^{4} w_{8}^{\prime 2}+w_{8}^{2} w_{8}^{\prime 4}\right) w_{7}^{2}\right\}\right)\left[w_{8}^{2}\right]\right)\left[w_{8}^{\prime 2}\right], \\
E_{2 p^{4}}(B \operatorname{Spin}(9)) \\
\simeq\left(E_{2 p^{3}}(B \operatorname{Spin}(7))\right. \\
\oplus\left(B\left(2, v_{1}, v_{2}\right)\left\{w_{16}\right\} \oplus B\left\{2 w_{4} w_{16}\right\}\right. \\
\oplus B\left(2, v_{1}\right)\left\{w_{8} w_{16}\right\} \oplus B\left\{2 w_{4} w_{8} w_{16}\right\} \\
\left.\left.\oplus B /\left(2, v_{1}, v_{2}, v_{3}, v_{4}\right)\left[w_{7}^{2}\right]\left\{w_{16}^{2} w_{8}^{2} w_{7}^{2}\right\}\right)\left[w_{8}^{2}\right]\right)\left[w_{16}^{2}\right] .
\end{aligned}
$$

Here we note $Q_{4} Q_{3}\left(w_{8} w_{8}^{\prime}\right)=\left(w_{8}^{4} w_{8}^{\prime 2}+w_{8}^{4} w_{8}^{\prime 2}\right) w_{7}^{2}$ and $Q_{4} Q_{3} w_{16}=w_{8}^{2} w_{7}^{2} w_{16}^{2}$. Hence $E_{2 p^{4}} \simeq E_{\infty}$ and the properties (1)-(3) and (7) hold.

The cohomology is $H^{*}\left(B F_{4} ; Z / 2\right) \simeq H^{*}\left(B G_{2} ; Z / 2\right) \otimes Z / 2\left[x_{16}, x_{24}\right]$. Moreover, $i^{*}: H^{*}\left(B F_{4} ; Z / 2\right) \rightarrow H^{*}(B \operatorname{Spin}(9) ; Z / 2)$ is injective with $i^{*} x_{16}=w_{16}+$ $\cdots$ and $i^{*} x_{24}=w_{8}^{3}+\cdots$. We can see that $B P^{*}\left(B F_{4}\right)$ has the similar form as $B P^{*}(B \operatorname{Spin}(9))$ by exchanging $w_{8}$ for $x_{24}$. Therefore the properties (1)-(3), (7) hold for $G=F_{4}$.

The cohomology of $B \operatorname{Spin}(10)$ and $E_{6}$ are

$$
\begin{aligned}
& H^{*}(B \operatorname{Spin}(10) ; Z / 2) \\
& \quad \simeq H^{*}(B \operatorname{Spin}(7) ; Z / 2) \otimes Z / 2\left[w_{10}, w_{32}^{\prime}\right] /\left(w_{7} w_{10}\right),
\end{aligned}
$$

$$
H^{*}\left(B E_{6} ; Z / 2\right) \simeq H^{*}(B \operatorname{Spin}(7) ; Z / 2) \otimes Z / 2\left[w_{10}, y_{18}, w_{32}^{\prime}, y_{34}, y_{48}^{\prime}\right] / R,
$$

where $R$ is the relation given Theorem 6.21 in [K-M2]. Since $Q_{n} w_{10}=0$ and $Q_{j} w_{32}^{\prime}=0$ for $0 \leq j \leq 3$ from Theorem 6.7 in [Q2], we get

$$
\begin{aligned}
E_{2 p^{3}}( & B \operatorname{Spin}(10) ; Z / 2) \\
\quad & \simeq\left(E_{2 p^{3}}(B \operatorname{Spin}(7)) \oplus B P^{*}\left[w_{6}, w_{8}, w_{10}\right]\left\{w_{10}\right\}\right) \otimes Z_{(2)}\left[w_{32}^{\prime}\right] .
\end{aligned}
$$

By the similar reason, we get (6.18)

$$
\begin{aligned}
E_{2 p^{3}}\left(E_{6} ; Z / 2\right) \simeq & \left(E_{2 p^{3}}(B \operatorname{Spin}(7)) \oplus B P^{*}\left[w_{6}, w_{8}, w_{10}, y_{18}\right]\left\{w_{10}, y_{18}, y_{34}\right\}\right) \\
& \otimes Z_{(2)}\left[w_{32}^{\prime}, w_{48}^{\prime}\right] .
\end{aligned}
$$

Therefore $E_{2 p^{3}} \simeq E_{\infty}$ and the properties (1)-(3) and (7) hold for $G=\operatorname{Spin}(10)$ and $E_{6}$.

At last we consider the case $G=P S U(4 n+2)$. The cohomology is known from [K-M1]

$$
H^{*}(B P U(4 n+2) ; Z / 2) \simeq Z / 2\left[a_{2}, a_{3}, x_{8 k}, y(I)\right] / R
$$


where $1<k \leq 2 n, I=\left(i_{1}, \ldots, i_{r}\right)$ for $1<i_{1}<\cdots<i_{r} \leq 2 n+1$, $\left|y_{I}\right|=4 \sum i_{s}-2$, and $R$ is the ideal generated by $a_{3} y(I)$ and $y(I)^{2}+\cdots$ and $y(I) y(J)$.

From Theorem 6.10 in [K-M1], $x_{8 k}$ is the $4 k$ th Chern class of representation to $U\left(\left(\begin{array}{c}4 n+2 \\ 2\end{array}\right)\right)$. Hence $Q_{m} x_{8 k}=0$ for all $m \geq 0$. Note that

$$
0=Q_{m}\left(a_{3} y_{I}\right)=a_{2}^{*} a_{3}^{*} y_{I}+a_{3} Q_{m}\left(y_{I}\right)=a_{3} Q_{m}\left(y_{I}\right) \text {. }
$$

The $\operatorname{ker} \cdot a_{3}$ is generated by $(y(I))$, which is even dimensionally generated. Hence $Q_{m}\left(y_{I}\right)=0$, for all $m \geq 0$. Therefore

$$
E_{2 p}(B P U(4 n+2)) \simeq E_{2 p}(B S O(3)) \otimes Z_{(2)}\left[x_{8 k}\right] \oplus B P^{*}\left[a_{2}, x_{8 k}\right]\{y(I)\} .
$$

Hence (1)-(3), (7) hold also for these cases. A similar result holds for $G=$ $P S p(2 n+1)$ by using the result $[\mathrm{K}]$.

Adams conjectured that for all connected compact Lie group $G$, the map $r$ in (3.2) is injective for $p \geq 3$

$$
r: H^{*}(B G ; Z / p) \hookrightarrow{\underset{V}{\leftrightarrows}}_{\lim ^{*}} H^{*}(B V ; Z / p) .
$$

When $p=2$ the above $r$ is injective for $G=\operatorname{Spin}(8 n+k), k \equiv 1,7,8$ mod 8 by Quillen [Q] and for $G=S O(n), O(n), G_{2}, F_{4}$ by Borel [B] and for $G=E_{6}$ by Kono and Mimura [K-M2]. However for $G=E_{7}$ and $\operatorname{Spin}(11)$ the map $r$ is not epic. The cohomology of $B \operatorname{Spin}(11)$ is given by

$$
H^{*}(B \operatorname{Spin}(11) ; Z / 2) \simeq Z / 2\left[w_{4}, w_{6}, w_{7}, w_{8}, w_{10}, w_{11}\right] / R \otimes Z / 2\left[w_{64}\right]
$$

where $R=\left(w_{11} w_{6}+w_{10} w_{7}, w_{11}^{3}+w_{11}^{2} w_{4} w_{7}+w_{11} w_{8} w_{7}^{2}\right)$. Put

$$
x=w_{10}^{2} w_{11}+w_{10}^{2} w_{4} w_{7}+w_{10} w_{8} w_{7} w_{6} \in H^{31}(B \operatorname{Spin}(11) ; Z / 2) .
$$

Then

$$
w_{11}^{2} x=w_{10}^{2}\left(w_{11}^{3}+w_{11}^{2} w_{4} w_{7}+w_{11} w_{8} w_{7}^{2}\right)=0 .
$$

Note that $x=w_{11}\left(w_{10}^{2}+w_{10} w_{6} w_{4}+w_{8} w_{6}^{2}\right)$ and hence $x^{3}=0$. On the other hand, define a map $\phi: H^{*}(B \operatorname{Spin}(11) ; Z / 2) \rightarrow Z / 2\left[a_{10}, a_{11}\right] /\left(a_{11}^{3}\right)$ by $\phi\left(w_{j}\right)=$ 0 for $j=4,6,7,8,64$ and by $\phi\left(w_{10}\right)=a_{10}, \phi\left(w_{11}\right)=a_{11}$. This map is a ring homomorphism and $\phi(x)=a_{10}^{2} a_{11}, \phi(x)^{2} \neq 0$. Therefore $x^{2} \neq 0$ but $x^{3}=0$. Hence $r(x)=0$.

Lemma 6.22. If (6.21) holds, then Conjecture 5 holds, that is, for all odd dimensional elements $x \in H^{*}(B G ; Z / p)$, there are $i$ such that $Q_{m} x \neq 0$ for all $m \geq i$.

Proof. The $Q_{m}$-homology $H\left(H^{*}(B V ; Z / p) ; Q_{m}\right) \simeq \otimes Z / p\left[y_{i}\right] /\left(y_{i}^{p^{m}}\right),\left|y_{i}\right|=2$ from Künneth formula. If $|x| \leq\left|Q_{m}\right|=2\left(p^{m}-1\right)$, then $x \notin \operatorname{Im} Q_{m}$ and so $Q_{m} x \neq 0$.

Corollary 6.23. If (6.21) holds, e.g., $G=S O(n)$, then

$$
\rho\left(P(n)^{*}(B G) \rightarrow H^{*}(X ; Z / p)\right) \subset H^{\text {even }}(B G ; Z / p),
$$

for all $n \geq-1$ (where $P(-1)=B P)$.

Remark 6.24. All examples given in $\S \S 5$ and 6 satisfy the following conjecture stated in [T-Y]

$$
B P\langle n-1\rangle^{*}(B G) \simeq B P\langle n-1\rangle^{*} \otimes_{B P^{*}} B P^{*}(B G) \text { if } \operatorname{rank}_{p} G \leq n .
$$




\section{REFERENCES}

[B-N] M. Bakuradze and R. Nadiradze, Cohomological realizations of two-valued formal groups and their applications, Bull. Acad. Sci. Georgian SSR 128 (1987), 21-24.

[B] A. Borel, Sur l'homologie et la cohomologie die groupes de Lie compacts connexes, Amer. J. Math. 76 (1954), 273-342.

[H-K-R] M. Hopkins, N. Kuhn, and D. Revenel, Generalized group characters and complex oriented cohomology theories, preprint.

[H] J. Hunton, The Morava K-theories of wreath products, Math. Proc. Cambridge Philos. Soc. 107 (1990), 309-318.

[I] K. Inoue, The Brown-Peterson cohomology of BSO(6) , J. Kyoto Univ. 32 (1992), 655-666.

[J-W] D. Johnson and W. S. Wilson, BP-operations and Morava's extraordinary $K$-theories, Math. Z. 144 (1975), 55-75.

[K-M1] A. Kono and M. Mimura, On the cohomology of the classifying space of PSU $(4 n+2)$ and $P O(4 n+2)$, Publ. Res. Inst. Math. Sci. Kyoto Univ. 10 (1975), 691-720.

[K-M2] _ Cohomology mod 2 of the classifying space of compact connected Lie group of type $E_{6}$, J. Pure Appl. Algebra 6 (1975), 61-81.

[K-M-S] A. Kono, M. Mimura, and N. Shimada, Cohomology of classifying space of certain associative $H$-space, J. Math. Kyoto Univ. 15 (1975), 607-617.

[K] A. Kono, On cohomology mod 2 of classifying spaces of non-simply connected classical Lie groups, J. Math. Soc. Japan 27 (1975), 281-288.

[L1] P. Landweber, Coherence, flatness and cobordism of classifying spaces, Proc. Aarhus Summer Inst. on Algebraic Topology, 1970, pp. 256-269.

[L2] - Homological properties of comodules over $M U_{*}(M U)$ and $B P_{*}(B P)$, Amer. J. Math. 98 (1976), 591-610.

[L3] _-, Elements of infinite filtration in complex cobordism, Math. Scand. 30 (1972), 223-226.

[Q1] D. Quillen, The spectrum of an equivariant cohomology ring. I, II, Ann. of Math. (2) 94 (1971), 549-572, 573-602.

[Q2] _ The mod 2 cohomology rings of extra-special 2-groups and spinor groups, Math. Ann. 194 (1971), 197-212.

[T-Y] M. Tezuka and N. Yagita, Cohomology of finite groups and the Brown-Peterson cohomology. I, II, Lecture Notes in Math., vols. 1370, 1418, Springer-Verlag, Berlin and New York, 1989,1990 , pp. 396-408, 57-69.

[T1] H. Toda, Cohomology mod 3 of the classifying space $B F_{4}$ of the exceptional group $F_{4}$, J. Math. Kyoto Univ. 13 (1973), 97-115.

[T2] _ Cohomology of classifying spaces, Homotopy Theory and Related Topics, Adv. Stud. Pure Math., vol. 9, Academic Press, Boston, Mass., 1986, pp. 75-108.

[W] W. S. Wilson, The complex cobordism of $B O_{n}$, J. London Math. Soc. 29 (1984), 352-366.

[Y1] N. Yagita, On relations between Brown-Peterson cohomology and the ordinary $\bmod p$ cohomology theory, Kodai Math. J. 7 (1984), 273-285.

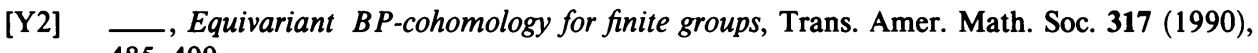
485-499.

Department of Mathematics, University of Aberdeen, Aberdeen AB9 2TY, United KINGDOM

Current address: Department of Mathematics, Kyoto University, Kitashirakawa Sakyoku, Kyoto, Japan

Department of Mathematics, Musashi Institute of Technology, Tamazutumi Setagayaku, Tokyo, Japan

Current address: Faculty of Education, Ibaraki University, Mito, Ibaraki-Ken, Japan 